\title{
AVALIAÇÃO DA EQUIVALÊNCIA FARMACÊUTICA EM COMPRIMIDOS DE HIDROCLOROTIAZIDA FABRICADOS POR COMPRESSÃO DIRETA E GRANULAÇÃO VIA ÚMIDA
}

Evaluation of pharmaceutical equivalence in hydrochlorothiazide tablets Manufactured In By Compression Direct And Grain Via Moist

Jonathann Corrêa Possato ${ }^{1}$, Edson de Oliveira Zaldguer ${ }^{1}$, Rahisa Scussel ${ }^{1}$, Ricardo Andrez Machado de Avila ${ }^{1}$, Eduardo João Agnes ${ }^{1}$

${ }^{1}$ Laboratório de Biologia Molecular e Celular, Unidade Acadêmica de Ciências da Saúde, Universidade do Extremo Sul Catarinense

Endereço para correspondência:

Jonathann Corrêa Possato

Laboratório de Biologia Celular e Molecular, Universidade do Extremo Sul Catarinense

Av. Universitária, 1105, Bairro Universitário, Criciúma - SC

CEP - 88806-000

Email: jcpossato@unesc.net 


\title{
Resumo
}

Objetivos: Avaliar a equivalência farmacêutica dos comprimidos de hidroclotiaziada de 50 miligramas produzidos por via direta e por via úmida, fabricados em aula do curso de farmácia na Universidade do Extremo Sul Catarinense. Metodologia: Foi realizado teste para a avaliação da velocidade de dissolução dos comprimidos de hidroclorotiazida. $O$ teste foi realizado seguindo parâmetros estabelecidos pela farmacopeia brasileira. Resultados: Submetemos os comprimidos testes ao aparelho de dissolutor, para o hidroclotiazida referência (Clorana) obtivemos valores de dissolução iguais a 96,4\% em 30 minutos, para comprimidos produzidos em aula por via direta, valores iguais a $71,2 \%$ em 30 minutos e produzidos por via úmida, valores iguais a 110,9\% em 30 minutos. Como exigido pela farmacopeia, os valores para a dissolução são acima de $60 \%$ da substância dissolvida em 30 minutos. Como preconizado na monografia da substância, os valores da concentração do analíto deve ser $93 \%$ a $107 \%$ da quantidade declarada. Neste quesito, só o referência obteve resultado satisfatório com valor de $101,9 \%$ da substância, quando por via direta teve valor de $75,6 \%$ e via úmida em $115,5 \%$ da substância declarada. Conclusão: O processo de fabricação de um fármaco pode causar uma alteração na sua disponibilidade, como testado in vitro pelo teste de dissolução. Para os testes observamos que não alcançamos os parâmetros exigidos pela monografia da substância.

Palavras-chaves: Equivalência Farmacêutica; Dissolução; Hidroclorotiazida.

\begin{abstract}
Objectives: To evaluate the equivalence of pharmaceutical 50 milligrams hydrochlorothiazide tablets produced by the direct pathway and wet, manufactured in the classroom in the pharmacy course at the Universidade do Extremo Sul Catarinense. Method: Test for assessment of the rate of dissolution of hydrochlorothiazide tablets were performed. The test was performed according to standards established by the Brazilian Pharmacopoeia. Results: tablets submitted the test appliance to the dissolutor to reference hydrochlorothiazide (Clorana) obtained values equal to $96.4 \%$ dissolution in 30 minutes, to tablets produced by direct route class, values equal to $71.2 \%$ in 30 minutes and produced via wet, values
\end{abstract}




\section{Artigo Original}

\section{Tecnologias em Saúde}

equal to $110.9 \%$ in 30 minutes. As required by the Pharmacopoeia the values for dissolution are above $60 \%$ of the dissolved substance in 30 minutes. As recommended in the monograph substantiates the values of the analyte concentration should be $93 \%$ to $107 \%$ of the labeled amount. In this regard references only got satisfactory result with a value of $101.9 \%$ of the substance, as by direct approach had value of $75.6 \%$ and $115.5 \%$ wet on the substance declared. Conclusion: The manufacturing process of a drug may cause a change in its availability, as tested by in vitro dissolution test. For testing we noticed that we did not reach the parameters required by the monograph substance.

Keywords: Pharmaceutical Equivalence; Dissolution; Hydrochlorothiazide.

\section{INTRODUÇÃO}

A via de administração de um fármaco mais comumente utilizada é a via oral. Dentre os fármacos administrados encontra-se a forma farmacêutica sólida, sendo comprimido o mais utilizado para terapêutica. A produção de comprimidos baseia-se nos processos compressão via granulação úmida, granulação seca e compressão direta ${ }^{1,2}$. A compressão de comprimidos leva aos rearranjos das partículas de resistência específica definida em um pelete, acarretando na deformação de partículas, formação de ligação entre as partículas e recuperação elástica por meio da ejeção do compactado a partir da matriz. A interação física durante a compressão de comprimidos são as interações intermoleculares no processo de mistura de pó. Devido à natureza termodinâmica das interações intermoleculares e interparticulares, é mais fácil entendê-las em termos de energia. No entanto, são as forças de interação entre corpos macroscópicos que muitas vezes são mais fáceis de medir experimentalmente ${ }^{3}$.

O processo de granulação consiste que partículas pulvéreas primárias se aglomerem de modo a formar partículas maiores, chamadas de grânulos. Em sua grande maioria, ela varia entre 0,2 e 0,5 mm, usados para produção de cápsulas ou comprimidos. A granulação é utilizada para prevenir segregação dos constituintes de uma mistura, assim não ocorre depósito de partículas mais densas na base do 


\section{Artigo Original}

\section{Tecnologias em Saúde}

recipiente, obtendo-se uma mistura mais homogênea, pois cada grânulo terá proporção correta de cada componente ${ }^{1}$.

A granulação úmida, por sua vez, implica na formação de uma massa da mistura utilizando-se líquido de granulação, o qual deve ser volátil, atóxico e facilmente removido após secagem. Este líquido ainda pode ser usado isoladamente, empregado com água, etanol ou isopropanolol, ou como também pode ser utilizado em combinação com agentes aglutinantes já dissolvidos, a fim de garantir a adesão após a secagem ${ }^{1}$.

Diferente da granulação úmida, a compressão direta é mais simples, participando de duas etapas: mistura e compressão. Esse processo vem ganhando espaço pela simplicidade e menor tempo de processo produtivo, o qual é mais viável em termos econômicos. Porém, para desenvolvimento desta técnica deve-se ater às características da matéria-prima e dos seus excipientes, visando a otimização dos processos de mistura, fluidez e compressibilidade do pó. Em alguns casos a granulação úmida ainda é a melhor alternativa, pois elimina alguns dos problemas da compressão direta, a exemplo a segregação do pó ${ }^{1,4}$.

A Hidroclorotiazida (Figura 1) é um diurético da classe das benzotiazidas, amplamente utilizada como diurético, adjuvante a tratamento da hipertensão arterial e na redução de edema associado à insuficiência cardíaca congestiva. Sua administração é por via oral em doses de 25 e $50 \mathrm{mg}$ na forma farmacêutica de comprimidos $^{5}$.

Figura 1. Estrutura química da hidroclorotiazida.

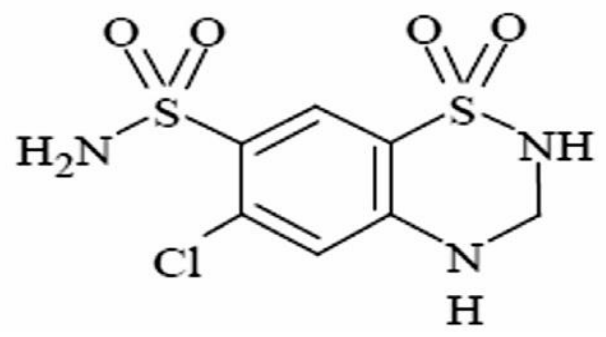

Segundo a resolução no 899 de 2003, deve-se demonstrar que o método é apropriado para a finalidade pretendida, ou seja, a determinação qualitativa, semiquantitativa e/ou quantitativa de fármacos e outras substâncias. No caso de 


\section{Artigo Original \\ Tecnologias em Saúde}

metodologia analítica descrita em farmacopeias, essa é devidamente reconhecida pela ANVISA, a metodologia é considerada validada ${ }^{6}$.

O teste de dissolução possibilita determinar quantidade de fármaco dissolvida, quando exposto a metodologia descrita nas monografias da substância em todas as condições, assim se enquadrando como teste de desempenho, categoria III pela resolução 899 de 20036,7. O presente estudo tem como objetivo avaliar o processo de fabricação dos comprimidos de hidroclotiazida, sendo por compressão direta e granulação úmida com mesma quantidade de analito $(50 \mathrm{mg})$ e seguindo a metodologia descrita na farmacopeia brasileira. Para isso foi realizado teste de dissolução, verificando se o comportamento se iguala a medicamento referência, no qual anteriormente foi submetido a testes mais precisos para sua liberação. Importante salientar também que o desenvolvimento deste trabalho justifica-se ao baixo fluxo do pó de hidroclorotiazida durante a fabricação de comprimidos.

\section{MATERIAIS E MÉTODOS}

\section{Materiais}

O presente estudo utilizou medicamento Hidroclorotiazida (Clorana, SanofiAventis farmacêutica Itda, São Paulo, Brasil), Hidroclorotiazida (Natural Pharma, Jin Jan Juyan chemicals), ácido clorídrico 37\% (Química Moderna), hidróxido de sódio (dinamica), dissolutor (Nova Ética), Espectrofotômetro (Femto).

\section{Amostra}

As amostras utilizadas foram comprimidos de clorana (hidroclotiaziada) 50 $\mathrm{mg}$ no lote: 314221 ; comprimidos de hidroclorotiazida de $50 \mathrm{mg}$ produzidos por via direta e via úmida por acadêmicos do curso de farmácia da Universidade do Extremo Sul Catarinense. 
Artigo Original

Tecnologias em Saúde

Compressão Direta

O processo de fabricação por via direta se faz pela pesagem da matériaprima, trituração, tamisação, mistura e compressão. Os excipientes foram pesados seguindo as proporções da Tabela 1, para o total de $100 \mathrm{~g}$ da mistura.

Tabela 1. Valores para formulação de $100 \mathrm{~g}$.

\begin{tabular}{cc}
\hline Matéria-Prima & Qtd em gramas \\
\hline Aerosil & $0,3 \mathrm{~g}$ \\
Talco & $20 \mathrm{~g}$ \\
Amido & $52,20 \mathrm{~g}$ \\
Estearato de magnésio & $2,5 \mathrm{~g}$ \\
Hidroclorotiazida & $20 \mathrm{~g}$ \\
\hline Total & $100 \mathrm{~g}$ \\
\hline
\end{tabular}

\section{Via Úmida}

Para o processo de fabricação por via úmida (Tabela 2), seguem-se as seguintes etapas: pesagem, trituração, tamisação, mistura, aglutinação, granulação, secagem, calibração, lubrificação e compressão. A diferença entre a compressão direta é a obtenção de uma massa úmida na etapa de aglutinação, para melhor uniformidade dos grânulos.

Tabela 2. Valores para formulação de 1200g.

\begin{tabular}{cc}
\hline Matéria-Prima & Quantidade \\
\hline Amido & $690 \mathrm{~g}$ \\
Talco & $150 \mathrm{~g}$ \\
Álcool Etílico & $300 \mathrm{~mL}$ \\
PVP K30 & $60 \mathrm{~g}$ \\
Hidroclorotiazida & $300 \mathrm{~g}$ \\
Total & $1200 \mathrm{~g}$ \\
\hline
\end{tabular}


A linearidade relaciona-se com a capacidade de uma metodologia analítica demonstrar que os resultados obtidos são diretamente proporcionais à concentração do analito de interesse na amostra, dentro de um intervalo especificado. Recomenda-se que seja determinada através de no mínimo cinco concentrações diferentes. Por meio do software Excel realizou-se os cálculos estatísticos e foi determinado o coeficiente de correlação que deve ser para fins de validação do método analítico maior ou igual a $0,99^{6}$.

\section{Teste de Dissolução}

O teste de dissolução foi realizado seguindo os parâmetros preconizados pela farmacopeia brasileira: Aparelhagem (cestos), $900 \mathrm{~mL}$ de ácido clorídrico $0,1 \mathrm{M}$ como meio de dissolução em velocidade de 100 rotações por minuto. A leitura foi realiza em espectrofotômetro em comprimento de onda $272 \mathrm{~nm}$, contendo uma tolerância de não menos que $60 \%$ do fármaco se dissolva em $30 \mathrm{~min}^{6,7}$.

\section{Perfil de Dissolução}

Para construção do perfil de dissolução foram coletadas alíquotas de $4 \mathrm{~mL}$ nos tempos 5, 10, 20, 30 e 45 minutos seguido de diluição de $1 \mathrm{~mL}$ da alíquota para $25 \mathrm{~mL}$ de água ${ }^{9}$. A leitura foi realizada em espectrofotômetro em comprimento de onda de $272 \mathrm{~nm}$, utilizando-se o diluente como branco. A curva foi construída por meio do método de doseamento, pesando-se $0,040 \mathrm{~g}$ de hidroclotiazida e diluindo em $100 \mathrm{~mL}$ de hidróxido de sódio $0,1 \mathrm{M}$ nas diferentes concentrações de $4 \mu \mathrm{g}, 8 \mu \mathrm{g}$, $12 \mu \mathrm{g}, 16 \mu \mathrm{g}$ e $20 \mu \mathrm{g}$. A partir das leituras obtidas das concentrações citadas foi realizada a curva de calibração e obtenção da equação de reta e seu respectivo coeficiente de correlação para avaliação da linearidade do método ${ }^{6,7}$. A comparação da dissolução dos comprimidos é obtida pelo cálculo do F2 (Equação 1), que corresponde a uma medida de semelhança entra as porcentagens dissolvidas de ambos os perfis, e F1 (Equação 2), que corresponde a diferença entre os perfis. 


$$
\begin{gathered}
\text { Equação } 1 . \\
F_{1}=\left\{\left[\sum_{i=1}^{n}|R f-T t|\right]\left[\sum_{i=1}^{n} R f\right]\right\} * 100
\end{gathered}
$$

\section{Equação 2.}

$$
F_{2}=50 \times \log \left\{\left[1+\left(\frac{1}{n}\right) \times \sum_{i=1}^{n}\left(R_{f}-T_{t}\right)^{2}\right]^{-0,5} \times 100\right\}
$$

Onde: $\mathrm{n}$ = número de tempos de coleta considerados para fins de cálculo; Rt = valor de porcentagem dissolvida no tempo $\mathrm{t}$ (obtido com medicamento referência); $\mathrm{Tt}=$ valor de porcentagem dissolvida do medicamento teste no tempo $t^{7,8,9}$.

Para que dois perfis de dissolução sejam considerados semelhantes o valor de $\mathrm{F} 1$ deve permanecer entre 0 e 15, sendo quanto mais próximo de 0 mais idêntico. E para o resultado de F2, deve estar entre 50 e 100, quanto mais próximo a 100 as curvas são idênticas ${ }^{9}$.

\section{RESULTADOS E DISCUSSÕES}

As leituras das soluções para curva de calibração obtiveram valor de correlação linear $R^{2}$ igual 0,9970 e/ou $R$ igual a 0,9988 , em $272 \mathrm{~nm}$, os quais atendem aos parâmetros da farmacopeia que indica $R$ igual ou maior que 0,980 para avaliação do método de trabalho em controle de medicamentos ${ }^{6}$. O coeficiente de correlação $(R)$ obtido também foi superior ao valor preconizado pela ANVISA, RDC 899 de 2003 para validação do método, que teria que ser superior ou igual a 0,99, indicando uma ótima linearidade e correlação entre a concentração do analito e a resposta do espectrofotômetro, como constata-se na Figura 2. 
Artigo Original

Tecnologias em Saúde

Figura 2. Gráfico da curva de calibração obtida para a hidroclorotiazida com sua respectiva equação de reta e coeficiente de correlação $\left(R^{2}\right)$.

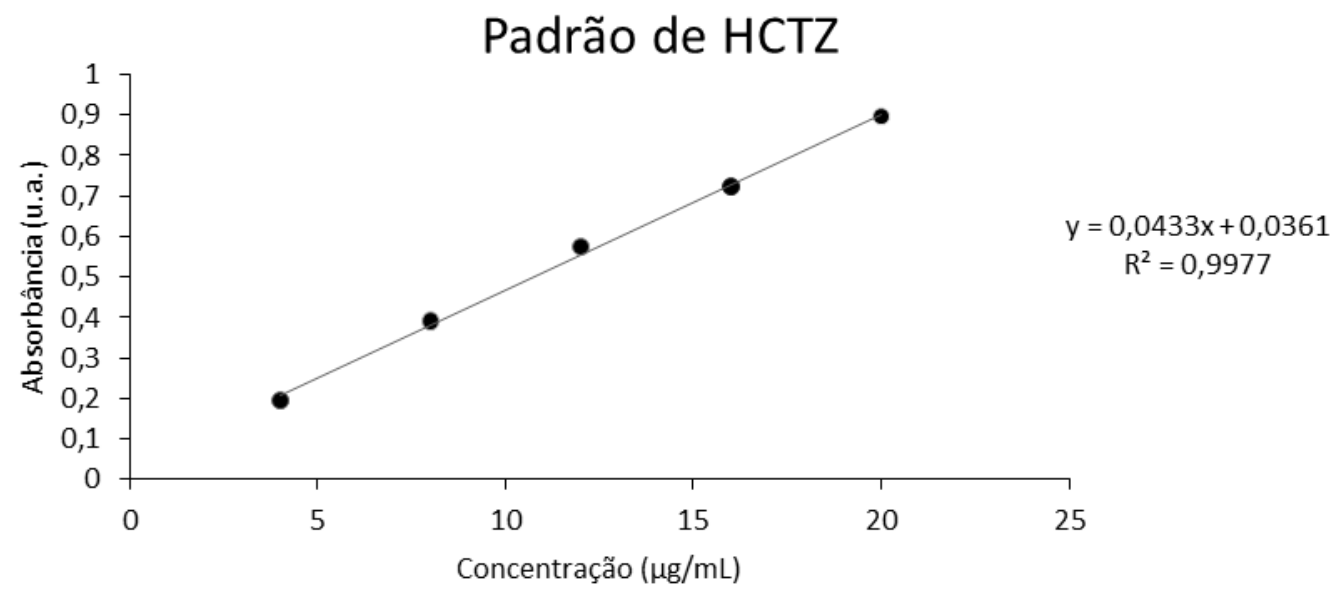

A partir da obtenção da curva de calibração, utilizou-se a equação de reta para os valores obtidos nas leituras do medicamento referência, os quais foram adicionados à equação como valor de $Y$, sendo $y=0,0433 x+0,0361$. Assim os valores obtidos foram corrigidos pelas diluições gerando valor em miligrama do medicamento, os resultados podem ser verificados na Tabela 3.

Tabela 3. Valores da dissolução do comprimido de Hidroclotiazida referência.

\begin{tabular}{cccccc}
\hline Clorana & 5 'min & $10^{\prime} \mathrm{min}$ & $20^{\prime} \mathrm{min}$ & 30 'min & $45^{\prime} \mathrm{min}$ \\
\hline Comprimido 1 (U.A) & 0,090 & 0,099 & 0,118 & 0,128 & 0,130 \\
Comprimido 2 (U.A) & 0,095 & 0,102 & 0,120 & 0,128 & 0,130 \\
Comprimido 3 (U.A) & 0,087 & 0,102 & 0,103 & 0,120 & 0,131 \\
Média & 0,091 & 0,101 & 0,114 & 0,125 & 0,130 \\
Concentração $\mu \mathrm{g} / \mathrm{ml} \mathrm{HCTZ}$ & 1,260 & 1,499 & 1,791 & 2,061 & 2,176 \\
Concentração mg de HCTZ & 29,489 & 35,073 & 41,918 & 48,223 & 50,925 \\
\% do fármaco & 59,0 & 70,1 & 83,8 & 96,4 & 101,9 \\
\hline
\end{tabular}

A porcentagem da concentração do hidroclorotiazida pode variar para o mínimo $93 \%$ e máximo de $107 \%$ da quantidade declarada ${ }^{8}$, neste caso $50 \mathrm{mg}$. 
Artigo Original

Tecnologias em Saúde

Podemos observar na Tabela 1 que o medicamento referência está dentro dos parâmetros. Nas Tabelas 4 e 5 encontram-se os valores dos comprimidos testes que, quando comparados aos parâmetros da farmacopeia, observamos que ele não se enquadra, mostrando valores abaixo do permitido para fabricação por via direta e acima do permitido para via úmida.

Entretanto, para todos os comprimidos testados observamos que ambos dissolveram quantidade maior que $60 \%$ da substância em 30 minutos, como preconizado pela farmacopeia.

Tabela 4. Valores da dissolução hidroclorotiazida por compressão direta.

\begin{tabular}{cccccc}
\hline Direta & 5 'min & $10^{\prime} \min$ & $20^{\prime} \min$ & $30^{\prime} \mathrm{min}$ & $45^{\prime} \mathrm{min}$ \\
\hline Comprimido 1 (U.A) & 0,081 & 0,083 & 0,095 & 0,096 & 0,095 \\
Comprimido 2 (U.A) & 0,095 & 0,092 & 0,096 & 0,098 & 0,110 \\
Comprimido 3 (U.A) & 0,071 & 0,097 & 0,106 & 0,112 & 0,113 \\
Média & 0,082 & 0,091 & 0,099 & 0,102 & 0,106 \\
Concentração $\mu \mathrm{g} / \mathrm{ml} \mathrm{HCTZ}$ & 1,068 & 1,260 & 1,453 & 1,522 & 1,614 \\
Concentração mg de HCTZ & 24,985 & 29,489 & 33,992 & 35,613 & 37,775 \\
\% do fármaco & 50,0 & 59,0 & 68,0 & 71,2 & 75,6
\end{tabular}

O valor abaixo, quando em compressão direta, pode ser pela diferença de fluxo do pó, causada por excipientes e/ou pela diferença do processo de fabricação. A diferença também pode ser erro no processo de pesagem da matéria-prima ${ }^{10}$.

Tabela 5. Valores da dissolução hidroclorotiazida por via úmida.

\begin{tabular}{cccccc}
\hline Via Úmida & 5 'min & 10 'min & $20^{\prime}$ min & $30^{\prime} \mathrm{min}$ & $45^{\prime} \mathrm{min}$ \\
\hline Comprimido 1 (U.A) & 0,102 & 0,116 & 0,130 & 0,138 & 0,142 \\
Comprimido 2 (U.A) & 0,112 & 0,120 & 0,132 & 0,139 & 0,146 \\
Comprimido 3 (U.A) & 0,100 & 0,125 & 0,131 & 0,139 & 0,141 \\
Média & 0,105 & 0,120 & 0,131 & 0,139 & 0,143 \\
Concentração $\mu \mathrm{g} / \mathrm{ml} \mathrm{HCTZ}$ & 1,584 & 1,945 & 2,192 & 2,369 & 2,469 \\
Concentração mg de HCTZ & 37,055 & 45,521 & 51,285 & 55,429 & 57,770 \\
\% do fármaco & 74,1 & 91,0 & 102,6 & 110,9 & 115,5 \\
\hline
\end{tabular}


Para via úmida podemos citar que o processo de fabricação está diretamente ligado ao fluxo do pó, auxiliando na mistura e na compressão do comprimido ${ }^{10}$. Na Figura 3 observamos os gráficos da dissolução dos medicamentos testados, sendo que quanto maior o tempo mais será dissolvido e maior concentração do fármaco estará no meio diluente.

Figura 3. Gráfico da dissolução dos comprimidos de Hidroclorotiazida referência, obtido por compressão direta e por compressão indireta (via úmida).

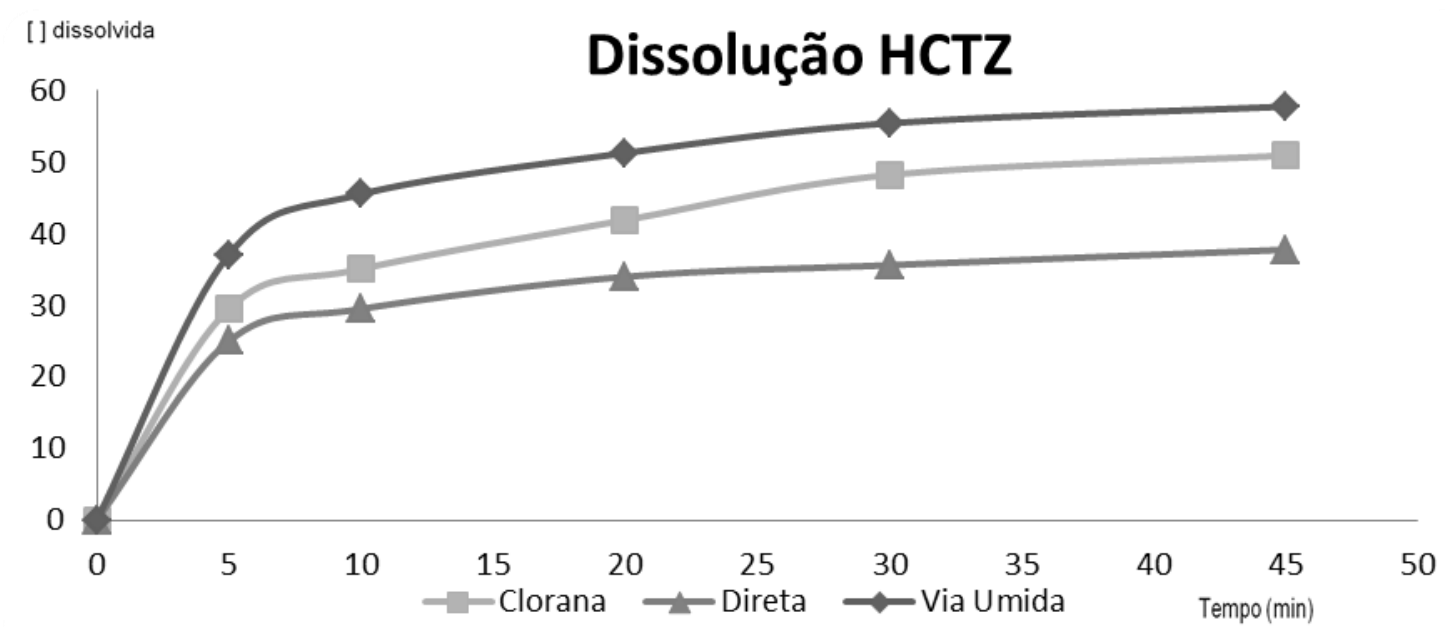

A comparação dos gráficos foi realizada pelo cálculo de $\mathrm{F} 1$, que demonstra a diferença entre os perfis de dissolução, e F2, que demonstra a semelhança destes perfis $^{7,9}$. A diferença calculada em $\mathrm{F} 1$ deve ficar entre 0 e 15, sendo que quanto mais próximo a 0 mais idênticas as dissoluções, e F2 deve ficar entre 50 e 100, sendo que quanto mais próximo de 100, maior a semelhança na dissolução dos comprimidos comparados. Como observa-se na Tabela 4, no cálculo obtivemos resultados não satisfatórios, sendo que os resultados de $F 1=20,28$ e $F 2=51,11$ para comprimido fabricado por via direta explanam que a semelhança entre a dissolução de tal comprimido em relação ao referência é baixa. O valor de F2 $(53,62)$ para comprimido fabricado por via úmida revela que este foi mais idêntico ao clorana quando comparado ao fabricado por via direta. Para F1 obtivemos os mesmos resultados em relação aos dois métodos de fabricação quando comparados ao Clorana. 
Tabela 6. Valores dos cálculos de semelhança (f2) e diferença (f1).

\begin{tabular}{lrr} 
& Clorana/Direta & Clorana/Via úmida \\
\hline F1 & 21,28 & $-20,15$ \\
F2 & 51,11 & 53,62 \\
\hline
\end{tabular}

Percebe-se que os métodos de fabricação testados tiveram comportamentos distintos em relação à sua velocidade de dissolução. Observou-se também que o processo de fabricação teve interferência na quantidade/homogeneidade do principio ativo no comprimido, como ilustra a Figura 3. Desta forma evidencia-se a importância do desenvolvimento da forma farmacêutica para sucesso terapêutico e comercial.

\section{CONCLUSÃO}

A comparação dos perfis de dissolução realizados neste trabalho revela que os processos de fabricação por via direta e via úmida obtiveram resultados não satisfatórios quando comparados com medicamento referência, uma vez que não atingiram todos os parâmetros preconizados. O presente estudo ainda evidencia a importância no processo de fabricação, já que cada detalhe pode interferir na sua ação farmacológica ou mesmo na própria fabricação, reduzindo fluxo do pó, por exemplo. A diferença na dissolução dos comprimidos estará diretamente ligada à sua ação, porque se o comprimido não dissolver e/ou dissolver rápido pode não ocorrer efeito farmacológico esperado, ou ainda se tornar tóxico ao organismo, respectivamente.

\section{REFERÊNCIAS}

1 - Alderborn G. Comprimidos e compressão. In: Aulton M. (ed.). Delineamento de formas farmacêuticas. 2. ed. Porto Alegre: Artmed; 2005. p. 403-6.

2 - Banker GS, Anderson NR. Comprimidos. In: Lachman L, Lieberman HA, Kanig JL. Teoria e prática na indústria farmacêutica. Lisboa: Fundação Calouste Gulbenkian, 2001. p.509-96. 
3 - Wang J, Wen H, Desai D. Lubrication in tablet formulations. Eur. J. Pharm. Biopharm. 2010;75(1):1-15.

4 - Wells J. Pré-formulação farmacêutica. In: Aulton M. (ed.). Delineamento de formas farmacêuticas. 2. ed. Porto Alegre: Artmed; 2005. p.125-48.

5 - Goodman LS, Gilman AG. As bases Farmacológicas da Terapêutica. Rio de Janeiro: Editora McGraw-Hill, 2007.

6 - Agência Nacional de Vigilância Sanitária (Brasil). Resolução oㅜ 899, de 29 de Maio De 2003. Guia para validação de métodos analíticos e bioanalíticos. Publicado no Diário Oficial da União. 2003.

7 - Agência Nacional de Vigilância Sanitária (Brasil). Farmacopeia Brasileira. 5 ed. Brasília: Agência Nacional de Vigilância Sanitária; 2010;1:66-70.

8 - Agência Nacional de Vigilância Sanitária (Brasil). Farmacopeia Brasileira. 5 ed. Brasília: Agência Nacional de Vigilância Sanitária; 2010;2:1035-37.

9 - Agência Nacional de Vigilância Sanitária (Brasil). Resolução no 31 , de 11 de Agosto de 2010. Dispõe sobre a realização dos Estudos de Equivalência Farmacêutica e de Perfil de Dissolução Comparativo. Publicado no Diário Oficial da União. 2010:36-8.

10 - Lamolha MA, Serra CHR. Avaliação das propriedades de fluxo dos granulados e dissolução de comprimidos de hidroclorotiazida $50 \mathrm{mg}$ obtidos por granulação úmida. Rev Bras Ciênc Farm. 2007;(43):435-46. 